\title{
Segmentation and Analysis of Anterior Lamina Cribrosa Surface using Non Local MRF and Metropolis Hasting Algorithm
}

\author{
Abhisha Mano, Assistant professor, Rajas Institute of Technology for Women, \\ Nagercoil.Email:abhizion1986@gmail.com
}

\begin{abstract}
The segmentation of anterior Lamina Cribrosa surface from the OCT image is an essential task for analysis of glaucomatous damage. A Bayesian method is used to segment LC surface whereas prior knowledge about shape and position of LC layer is obtained by the non local Markov Random field and K-means segmentation. The Metropolis-Hastings (MH) algorithm provides autocorrelation graph and distribution of samples from a probability distribution. By using this technique acceptance probability is calculated. Finally, the LC layer is analysed whether it is normal or abnormal. This technique provides an accuracy of $96.7 \%$
\end{abstract}

\section{Introduction}

The Lamina Cribrosa (LC) is used in the identification of glaucomatous injury. The Lamina Cribrosa (LC) is a eye layer which gives rise to retinal ganglion cell that extends to the brain with visual data. A boundary is formed by the Lamina Cribrosa in between the intraocular space and retrobulbar space with intraocular pressure and retrolaminar pressure respectively. In optical coherence tomography (OCT) optic nerve head ONH structures can be viewed clearly. Nowadays, spectral domain SD-OCT Enhanced depth imaging (EDI) was used to view the retinal structures. The first and final layer of Lamina Cribrosa are inner limiting membrane and retinal pigment epithelium respectively. The internal limiting membrane is used to diagnose various interface diseases, in the macular area. The pigmented layer nourishes retinal visual cells. The pressure of eye is measured in millimeters of mercury ( $\mathrm{mm} \mathrm{Hg}$ ). The normal eye has a pressure of 12-22 $\mathrm{mm} \mathrm{Hg}$. Eventhough the pressure is greater than normal range, and there is no symptoms of glaucoma it is called as ocular hypertension. If pressure is between $12 \mathrm{~mm} \mathrm{Hg}$ to $22 \mathrm{~mm} \mathrm{Hg}$ then the Lamina Cribrosa layer is in normal stage else it is in abnormal stage.

In method[1], automatic segmentation of slices of LC are done in 3D OCT imaging.BM4D algorithm performs grouping and filtering. A 4D transform will exploit the nonlocal correlation occurring among the pixels. By inverse transformation estimates are obtained for grouped data[8]. Otsu threshold method is used to identify the glaucoma from the disease[11].By artificial neural network, optic disc size and rim area is estimated[4].Here bruch's membrane is located using a deconvolution method. By solving MAP-MRF labeling problem, contour is formed, which is used in segmentation [5]. Many LC segmentation techniques [2],[3].[6],[7],[9],[10] and the structures are analysed [12][13][14][15][16][17] and explained. An approach for segmenting the retinal images using superpixel is proposed in paper[18]. An wavelet based segmentation method is applied for DNA fragment sizing in AFM images and for mammogram images [19][23]. An encryption techniques in images using mutation is presented in paper[21].An optimization based segmentation of brain images is presented in paper[20].Edge detection of microarray images by applying wavelet technique is presented in paper [22].

This paper explains a method for segmentation of Lamina Cribrosa surface. A surface evolution method with shape parameter is used. The shape is varied by Markov random field in an iterative manner. The parameters are computed using a Metropolis-Hastings algorithm and the surface is analysed whether it is normal or abnormal. 


\section{Proposed Method}

Optical coherence tomography (OCT) is taken as input and given to the layer segmentation step. The first and the final layer of the Lamina Cribrosa, inner limiting membrane and retinal pigment epithelium respectively are segmented in this step. Then preprocessing is done where the area of focus is determined. A Bayesian method is used to segment LC surface whose prior knowledge about shape and position of LC layer is obtained by the non local Markov Random field and K-means segmentation.

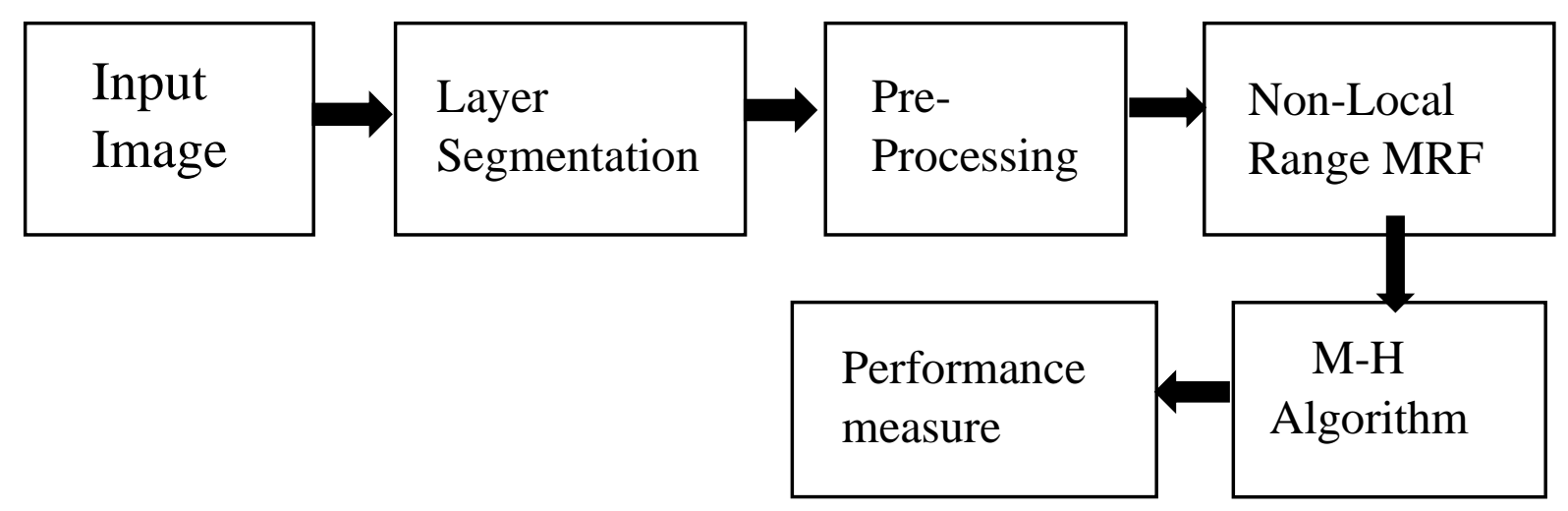

Fig: 1 Block Diagram of Proposed Method

\subsection{Non Local Markov Random Field Segmentation}

In non-local range MRF, the clique of each and every pixel has local neighbourhood patch whose topmost patches is obtained by block matching. The non local range of pixels are obtained from the image region based on similar patches. The filter that is applied over the non-local range is a 3-dimensional filter. Markov Random Field (MRF) will remove the correlation of intensities among voxels. The non local approach will remove the repeated structures in an image. The gradients of loss function are computed. Hence optimization based on gradients can be applied.

\subsection{Metropolis Hasting Algorithm}

Metropolis-Hastings algorithm a the sampling method is inspired by the Biased and Filtered Point Sampling (BFPS) method.

\section{Algorithm}

initialize $X^{(0)} \sim \mathrm{Q}(\mathrm{X})$ 


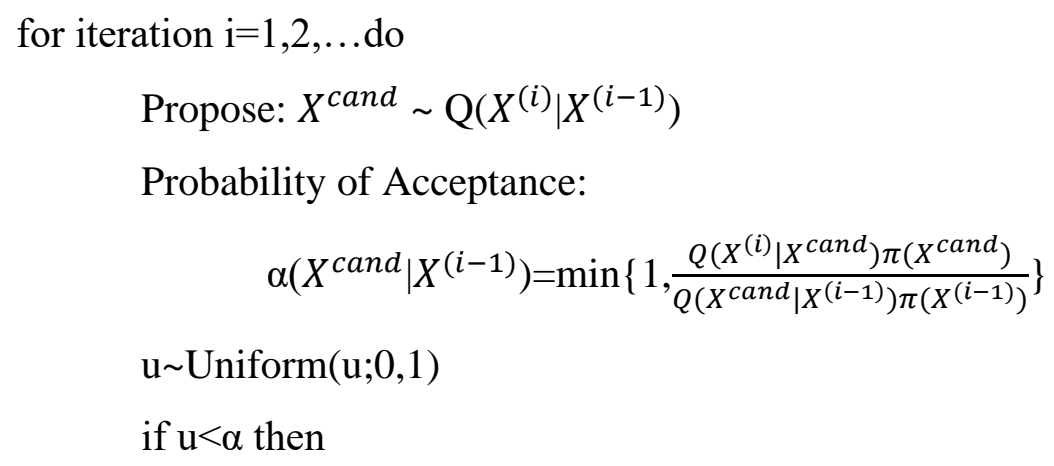

proposal gets accepted: $X^{i} \leftarrow X^{\text {cand }}$

else

proposal gets rejected: $X^{i} \leftarrow X^{(i-1)}$

end if

end for

First initialize the samples. Generate a proposal and compute the acceptance probability ,followed by accepting the candidate sample having a specified probability.

\section{Implementation}

Optical coherence tomography (OCT) image is taken and given as input to the system. Fig 2 shows the OCT image

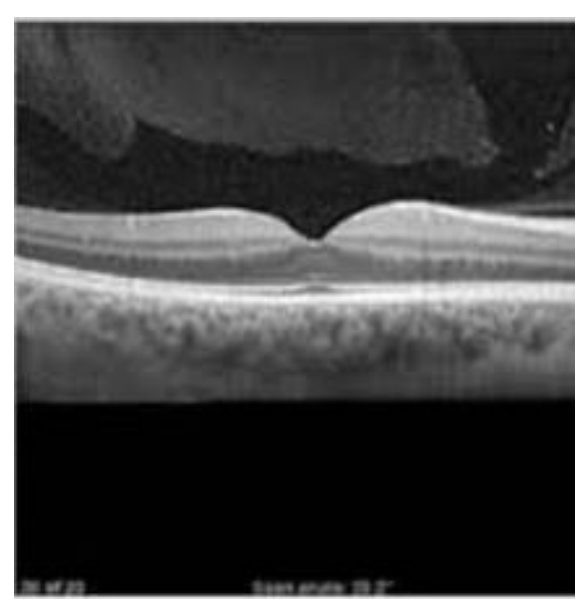

Fig 2. Input Image

The first layer of the Lamina Cribrosa i.e, inner limiting membrane and the final layer of the Lamina Cribrosa i.e, retinal pigment epithelium is segmented by the layer segmentation 
process. Fig 3 shows the segmented layers. The boundary of the Lamina Cribrosa layer is segmented using canny edge detection and the blurred image is obtain by using Gaussian blur. Fig $4 \mathrm{a}$ and Fig $4 \mathrm{~b}$ shows the boundary of image and blurred image respectively.

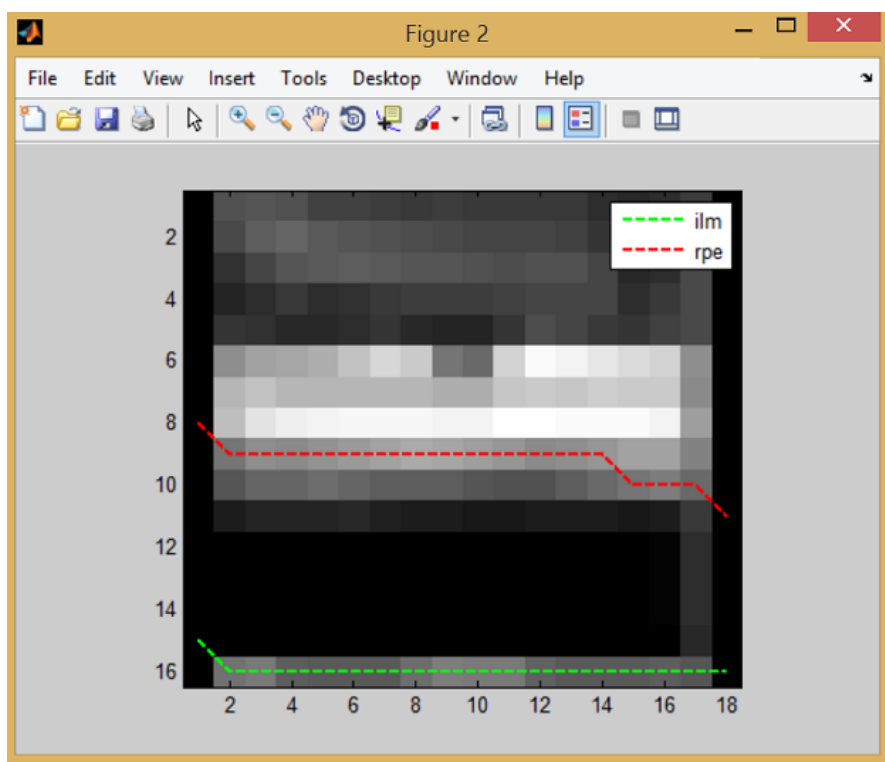

Fig 3 Segmented Layer

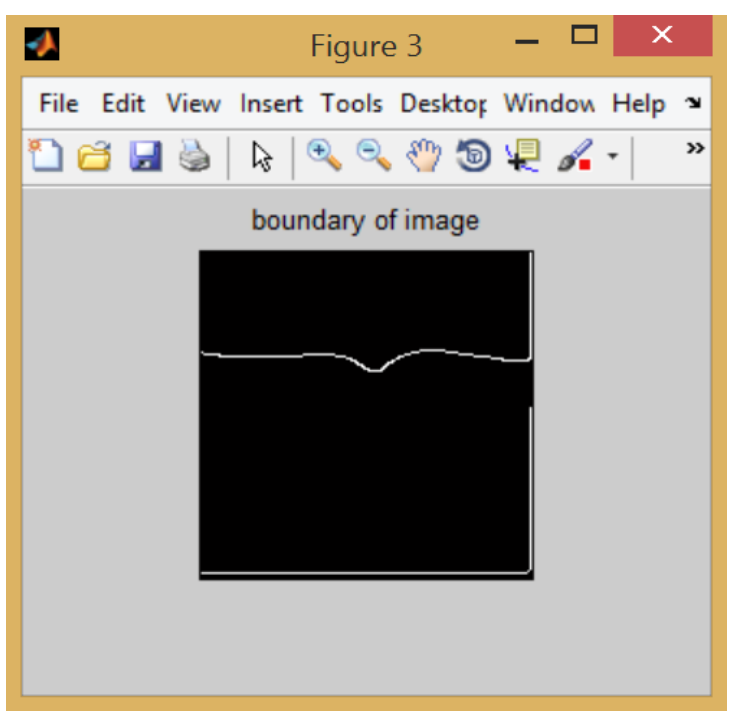

Fig 4a Boundary of the Image

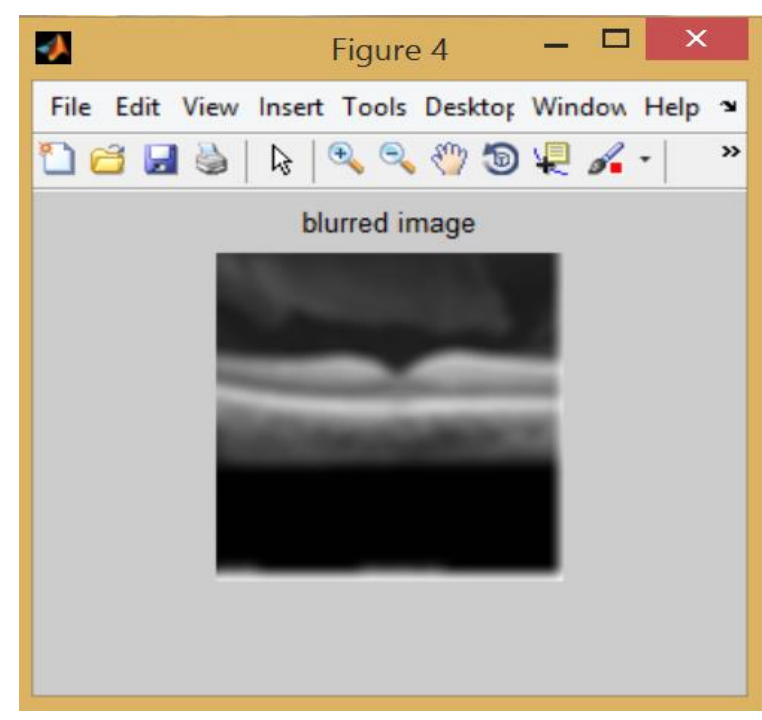

Fig 4b Blurred Image

From the prior knowledge of K-means segmentation initial label is obtained from which the final label can be obtained using Non local Markov random field segmentation. Figure 5 shows the initial label segmented output. 




Fig 5 K-means segmented output

Fig 6 shows the final label of the segmented output.

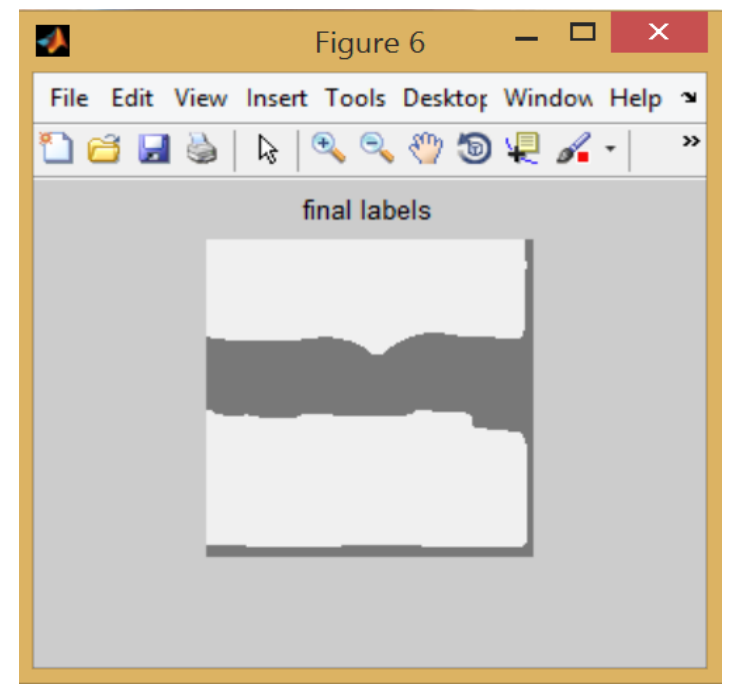

Fig 6 NLMRF segmented output

With the help of the initial and final labels, the density value of the Lamina Cribrosa is found. The density graph is shown in the Fig 7. The graph is plot between the EM iteration and the sum of $U$. Sum of $U$ denotes the value of initial labels and final labels which is obtained from the segmentation process. The value of EM iteration given as input is 10 . 


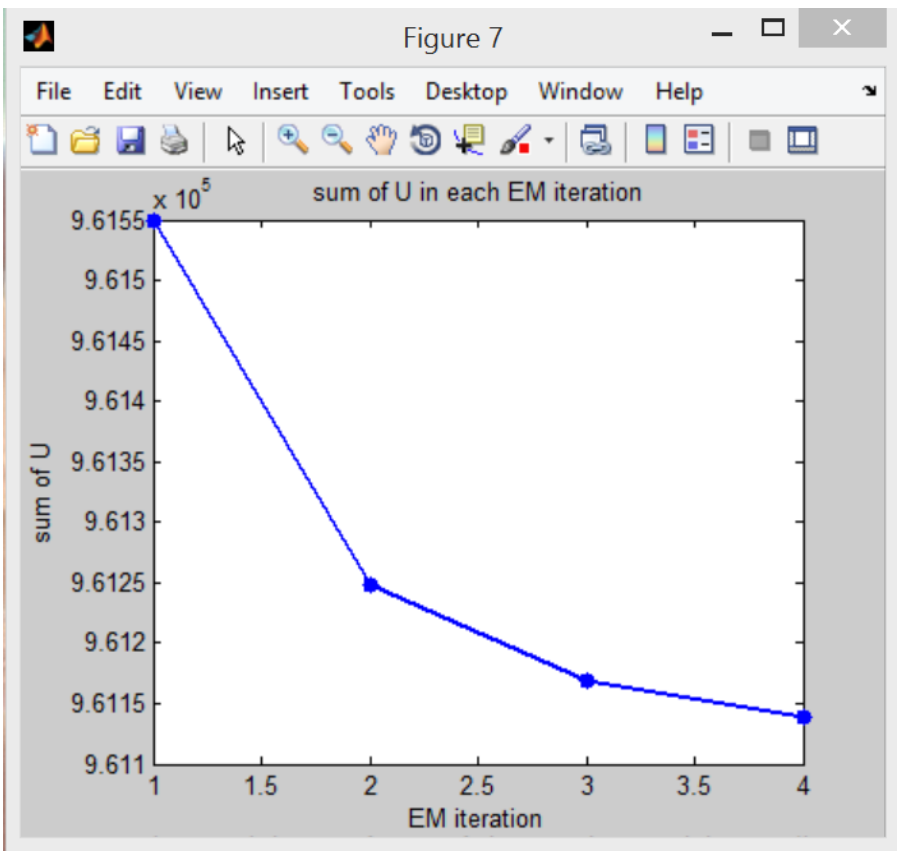

Fig 7 Density Graph

The Metropolis-Hastings (MH) algorithm provides autocorrelation graph and distribution of samples from a probability distribution. Here we choose 10000 samples for the process. The autocorrelation graph shows the values for the first 100 samples as well as last 100 samples.Fig 8 shows the autocorrelation graph obtained.

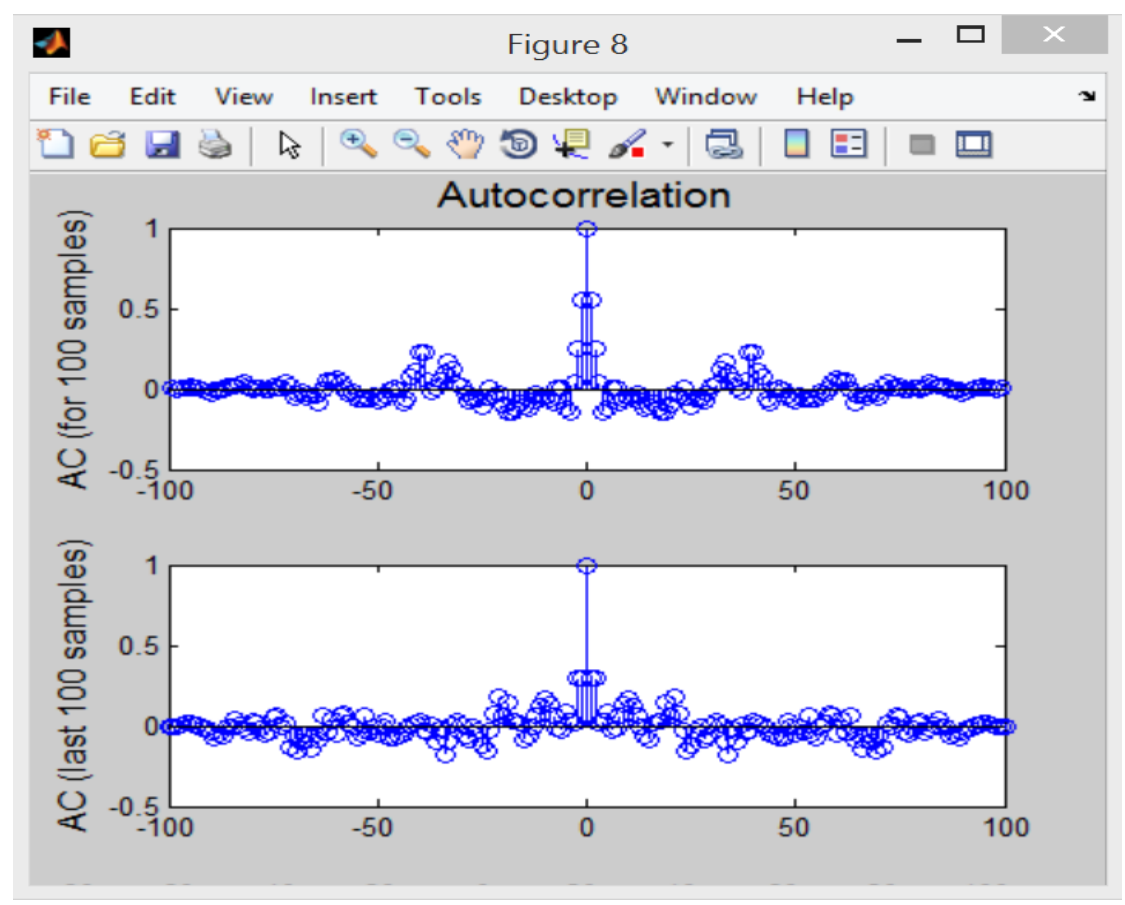

Fig 8 Autocorrelation Graph 
Fig 9 shows the distribution of samples graph. This graph is drawn with the help of probability density function. It provides the value of acceptance rate as 0.5418 .



Fig 9 Distribution of sample graph

With the help of the density, autocorrelation and the distribution of values the Lamina Cribrosa is verified whether it is in normal stage or in abnormal stage. If the value is below 12 $\mathrm{mm} \mathrm{Hg}$ then the help box will show as low pressure. If the value is above $22 \mathrm{~mm} \mathrm{Hg}$ then the help box will show as high pressure.

The value of pressure obtained from this image is 31.8256 . Thus the help box shows as high pressure.

\section{Conclusion}

The work proposed in this paper is a shape based surface evolution method for the segmentation of the anterior LC. A non local approach gives a MRF energy function. Metropolis-Hastings ( $\mathrm{MH}$ ) algorithm provides the accurate density, autocorrelation and distribution of values. The accurate value of pressure is obtained from which the Lamina Cribrosa is analysed whether it is normal or abnormal. Accuracy of this approach is $96.7 \%$ 


\section{REFERENCES}

[1] Nadler, Z., Wang, B., Wollstein, G., Nevins, J. E., Ishikawa, H., Kagemann, L., ... \& Liu, J. J. (2013). Automated lamina cribrosa microstructural segmentation in optical coherence tomography scans of healthy and glaucomatous eyes. Biomedical optics express, 4(11), 25962608.

[2] Burgoyne, C. F., Downs, J. C., Bellezza, A. J., Suh, J. K. F., \& Hart, R. T. (2005). The optic nerve head as a biomechanical structure: a new paradigm for understanding the role of IOPrelated stress and strain in the pathophysiology of glaucomatous optic nerve head damage. Progress in retinal and eye research, 24(1), 39-73.

[3] Lee, K., Niemeijer, M., Garvin, M. K., Kwon, Y. H., Sonka, M., \& Abramoff, M. D. (2009). Segmentation of the optic disc in 3-D OCT scans of the optic nerve head. IEEE transactions on medical imaging, 29(1), 159-168.

[4] Belghith, A., Bowd, C., Weinreb, R. N., \& Zangwill, L. M. (2014, August). A hierarchical framework for estimating neuroretinal rim area using 3D spectral domain optical coherence tomography (SD-OCT) optic nerve head (ONH) images of healthy and glaucoma eyes. In 2014 36th Annual International Conference of the IEEE Engineering in Medicine and Biology Society (pp. 3869-3872). IEEE.

[5] Chittajallu, D. R., Paragios, N., \& Kakadiaris, I. A. (2013). An explicit shape-constrained MRF-based contour evolution method for 2-D medical image segmentation. IEEE Journal of Biomedical and Health Informatics, 18(1), 120-129.

[6] Tovar-Vidales, T., Wordinger, R. J., \& Clark, A. F. (2016). Identification and localization of lamina cribrosa cells in the human optic nerve head. Experimental eye research, 147, 94-97. [7] Campbell, I. C., Coudrillier, B., Mensah, J., Abel, R. L., \& Ethier, C. R. (2015). Automated segmentation of the lamina cribrosa using Frangi's filter: a novel approach for rapid identification of tissue volume fraction and beam orientation in a trabeculated structure in the eye. Journal of The Royal Society Interface, 12(104), 20141009.

[8] Maggioni, M., Katkovnik, V., Egiazarian, K., \& Foi, A. (2012). Nonlocal transform-domain filter for volumetric data denoising and reconstruction. IEEE transactions on image processing, 22(1), 119-133.

[9] Jonas, J. B., Mardin, C. Y., Schlötzer-Schrehardt, U., \& Naumann, G. O. (1991). Morphometry of the human lamina cribrosa surface. Investigative ophthalmology \& visual science, 32(2), 401-405. 
[10] Quigley, H. A., \& Addicks, E. M. (1981). Regional differences in the structure of the lamina cribrosa and their relation to glaucomatous optic nerve damage. Archives of ophthalmology, 99(1), 137-143.

[11] Abe, R. Y., Gracitelli, C. P., Diniz-Filho, A., Tatham, A. J., \& Medeiros, F. A. (2015). Lamina cribrosa in glaucoma: diagnosis and monitoring. Current ophthalmology reports, 3(2), 74-84.

[12] Ram, S., Danford, F., Howerton, S., Rodríguez, J. J., \& Geest, J. P. V. (2017). Threedimensional segmentation of the ex-vivo anterior lamina cribrosa from second-harmonic imaging microscopy. IEEE Transactions on Biomedical Engineering, 65(7), 1617-1629.

[13] Cook, C., \& Foster, P. (2012). Epidemiology of glaucoma: what's new?. Canadian Journal of Ophthalmology, 47(3), 223-226.

[14] Rosenstein, R. E., \& Belforte, N. (2012). Radial optic neurotomy: a new surgical approach for glaucoma treatment?. Medical hypothesis, discovery and innovation in ophthalmology, $1(3), 52$.

[15] Yang, H., Qi, J., Hardin, C., Gardiner, S. K., Strouthidis, N. G., Fortune, B., \& Burgoyne, C. F. (2012). Spectral-domain optical coherence tomography enhanced depth imaging of the normal and glaucomatous nonhuman primate optic nerve head. Investigative ophthalmology \& visual science, 53(1), 394-405.

[16] Roberts, M. D., Sigal, I. A., Liang, Y., Burgoyne, C. F., \& Downs, J. C. (2010). Changes in the biomechanical response of the optic nerve head in early experimental glaucoma. Investigative ophthalmology \& visual science, 51(11), 5675-5684.

[17] Reis, A. S., O'Leary, N., Stanfield, M. J., Shuba, L. M., Nicolela, M. T., \& Chauhan, B. C. (2012). Laminar displacement and prelaminar tissue thickness change after glaucoma surgery imaged with optical coherence tomography. Investigative ophthalmology \& visual science, 53(9), 5819-5826.

[18] Mano, Abhisha (2020): CONTRAST ENHANCED SUPERPIXEL BASED SEGMENTATION OF RETINAL IMAGES. TechRxiv. Preprint. https://doi.org/10.36227/techrxiv.12254240.v1.2020

[19] Anand, S., Lakshmanan, B., Murugachandravel, J., Valarmathi, K., Mano, A., \& Kavitha, N. (2019). Wavelet-Based Automated DNA Sizing of Fragments through AFM Image Processing. International Journal of Engineering and Advanced Technology.8(5) .231-238.

[20] Mano, Abhisha (2020): A Novel Approach based on PSO Optimized K-Means in MRI Brain Image Segmentation. TechRxiv. Preprint. https://doi.org/10.36227/techrxiv.12310100.v1

[21] Premkumar, R., \& Anand, S. (2019). Secured and compound 3-D chaos image encryption using hybrid mutation and crossover operator. Multimedia Tools and Applications, 78(8), 9577-9593.

[22] Anand, S., \& Sindhuja, N. M. (2009, June). Spot edge detection in microarray images using balanced GHM multiwavelet. In 2009 International Conference on Control, Automation, Communication and Energy Conservation (pp. 1-4). IEEE. 
bioRxiv preprint doi: https://doi org/10.1101/2020.05.30.125682; this version posted June 1, 2020. The copyright holder has placed this preprint (which was not certified by peer review) in the Public Domain. It is no longer restricted by copyright. Anyone can legally share, reuse, remix, or adapt this material for any purpose without crediting the original authors.

[23] Muthukarthigadevi, R., \& Anand, S. (2013, February). Detection of architectural distortion in mammogram image using wavelet transform. In 2013 International Conference on Information Communication and Embedded Systems (ICICES) (pp. 638-643). IEEE. 